\title{
Clinical Results of Contralateral Arthroscopic Meniscectomy Performed with Unilateral Total Knee Arthroplasty: Minimum 3-year Follow-up
}

\author{
Sang Jin Lee, $\mathrm{MD}^{1}$, Bum-Sik Lee, $\mathrm{MD}^{2}$, Jong-Min Kim, $\mathrm{MD}^{3}$, and Seong-Il Bin, $\mathrm{MD}^{3}$ \\ ${ }^{1}$ Department of Orthopedic Surgery, Haeundae Paik Hospital, Inje University College of Medicine, Busan; ${ }^{2}$ Department of Orthopedic Surgery, Incheon St. Mary's \\ Hospital, The Catholic University of Korea College of Medicine, Incheon; ${ }^{3}$ Department of Orthopedic Surgery, Asan Medical Center, University of Ulsan College of \\ Medicine, Seoul, Korea
}

\begin{abstract}
Purpose: We assessed the clinical outcome of contralateral arthroscopic meniscectomy performed with unilateral total knee arthroplasty (TKA). Materials and Methods: From May 1999 to June 2006, 23 patients underwent unilateral total knee arthroplasty and contralateral arthroscopic meniscectomy at the same time. All patients were women and followed for at least 36 months, except 2 patients who died. For clinical assessment, range of motion of the knee joint, Hospital for Special Surgery (HSS) knee score and the Lysholm knee score were evaluated preoperatively and at the last follow-up. At arthroscopy, meniscal pathology and cartilage changes were recorded and classified according to the Outerbridge scale. Progression of osteoarthritis in the contralateral knee to subsequent TKA was also assessed.

Results: The mean age of the 21 patients was 67.1 years and the mean follow-up period was 5.7 years. All of the patients were diagnosed with osteoarthritis and had Outerbridge grade 3 or 4 cartilage changes. Eight of the 21 patients had subsequent TKA at an average of 3.1 years after the index operation. The other 13 patients had no further surgery and clinical results including the HSS knee score and the Lysholm score were improved from 74.5 and 60.6 preoperatively to 90.8 and 82.4 postoperatively, respectively $(\mathrm{p}<0.001)$.

Conclusions: Contralateral arthroscopic meniscectomy performed simultaneously with unilateral TKA produces relatively good results regardless of the presence of cartilage degeneration.
\end{abstract}

Keywords: Knee, Meniscus, Contralateral meniscectomy, Arthroplasty

\section{Introduction}

Total knee arthroplasty (TKA) has been well established as a successful treatment for end-stage knee osteoarthritis. Mostly, the patients are evaluated for radiographic evidence of osteoarthritis,

Received May 8, 2014; Revised (1st) September 1, 2014;

(2nd) October 15, 2014; Accepted October 28, 2014

Correspondence to: Seong-Il Bin, MD

Department of Orthopedic Surgery, Asan Medical Center, University of Ulsan College of Medicine, 88 Olympic-ro 43-gil, Songpa-gu, Seoul 138736, Korea

Tel: +82-2-3010-3528, Fax: +82-2-488-7877

E-mail: sibin@amc.seoul.kr

This is an Open Access article distributed under the terms of the Creative Commons Attribution Non-Commercial License (http://creativecommons.org/licenses/by-nc/4.0/) which permits unrestricted non-commercial use, distribution, and reproduction in any medium, provided the original work is properly cited. such as joint space narrowing and lower extremity malalignment to determine the necessity of TKA. For patients complaining of bilateral knee pain, either unilateral TKA on the more painful knee or simultaneous or consecutive bilateral TKA is performed according to the severity of radiographic osteoarthritis. Usually, radiographic evaluation of the former patients reveals severe osteoarthritis on one knee and mild osteoarthritic changes in the contralateral knee. Most of these patients are advanced in age and have some cartilage damage in the contralateral knee joint; therefore, they undergo unilateral TKA on the severe osteoarthritic knee and conservative treatment for the contralateral knee without further evaluation. Previous studies concerning the prognosis in the contralateral knee after primary unilateral TKA focused mainly on the observation or conservative treatment and showed that radiographic changes and symptomatic severity are associated with subsequent progression to arthroplasty in the contra- 
lateral knee $\mathrm{e}^{1-3)}$. However, if contralateral knee pain is severe from the beginning or has been exacerbated with time, meniscal lesion or cartilage damage might exist despite mild radiographic findings. If a meniscal tear is confirmed with magnetic resonance imaging and the symptoms are mainly from mechanical pain rather than osteoarthritic pain, surgical treatment such as meniscetomy might be considered. But, it is known that the prognosis of meniscectomy is closely associated with the cartilage status. In addition, considering that the loading of the contralateral knee joint generally increases in the early stage of rehabilitation after TKA, it is not clear whether the meniscectomy of the contralateral knee would be more beneficial than the conservative treatment. Until recently, there are few studies concerning the prognosis of meniscectomy of the contralateral knee after primary unilateral TKA for osteoarthritis.

In this study, we assessed the clinical outcomes of arthroscopic meniscectomy as well as the probability of a subsequent contralateral TKA when one knee was replaced due to osteoarthritis and the contralateral knee was treated with arthroscopic meniscectomy simultaneously under the hypothesis that contralateral arthroscopic meniscectomy performed with unilateral TKA would be effective regardless of cartilage degeneration.

\section{Materials and Methods}

Between 1999 and 2006, 729 primary TKAs were performed by the same surgeon at one institution. Of these, 23 patients who had both knee pain and underwent additional arthroscopic meniscectomy in the contralateral knee during TKA were included in this study. All of the patients were female and diagnosed with osteoarthritis of both knees. Institutional Review Board approval was obtained for this study.

Primary TKAs were performed using LPS or LPS-flex implant (NexGen; Zimmer Inc., Warsaw, IN, USA). Indications for surgery were varus thrust in walking and obliteration of the joint space on standing radiography. Arthroscopic meniscectomy were performed by the same surgeon based on the following indications: 1) mechanical meniscal pain was more predominant than the other osteoarthritic symptoms; 2) the meniscal signs such as McMurray test and joint line tenderness were clear; and 3) varus thrust in walking was not present ${ }^{4,5}$. Meniscectomy was not considered in cases where obliteration of the joint space was observed on standing radiography or the mechanical axis lay across the medial half of the medial tibial plateau of the whole lower limb on radiography. No additional procedures such as synovectomy, chondroplasty, and microfracture were performed.
Clinical assessment was performed using the medical records and telephone interviews. The range of motion (ROM), Hospital for Special Surgery (HSS) knee score, and the Lysholm knee score were evaluated preoperatively and at the last follow-up. At arthroscopy, all findings concerning meniscal pathology, ligament status, and cartilage changes were recorded on a special form. Cartilage changes were classified according to the Outerbridge scale $^{6}$. Also, we evaluated whether a contralateral TKA was subsequently performed and assessed the mean time from the initial meniscectomy to the subsequent TKA.

Statistical analysis was done using SPSS ver. 17.0 (SPSS Inc., Chicago, IL, USA). Preoperative ROM, knee scores and arthroscopic findings were compared between the two groups by the use of an independent t-test or a Fisher's exact test. Clinical results of group II were assessed using paired t-tests. Statistical significance was set at $\mathrm{p}<0.05$.

\section{Results}

All patients were followed for at least 36 months, except 2 patients who died within 12 months after the index surgery. The mean age of the 21 patients at operation was 67.1 years (range, 56 to 71 years) and the mean follow-up period was 5.7 years (range, 3.1 to 10.1 years).

At arthroscopic operation, all of the patients had Outerbridge grade III or IV cartilage changes on the medial femoral condyle or the medial tibial plateau. Eight patients had a horizontal tear of the posterior horn of the medial meniscus. Ten patients had a radial tear of the body or posterior horn of the medial meniscus. The other three patients had a complex tear of the medial meniscus, a complex tear of the lateral meniscus and a flap tear of the medial meniscus with a horizontal tear of the lateral meniscus, respectively. Partial meniscectomy of the medial meniscus was performed in 17 patients, subtotal meniscectomy of the medial meniscus in 2, partial meniscectomy of the lateral meniscus in 1, and subtotal meniscectomy of the medial meniscus and partial meniscectomy of the lateral meniscus in 1 (Table 1).

Of the 20 patients who had partial or subtotal meniscectomy of the medial meniscus, 8 had a subsequent TKA surgery at an average of 3.1 years (range, 2.0 to 6.6 years) after initial operation (group I). Including two patients who had subtotal meniscectomy, 13 of the 21 patients had no further surgery (group II). The mean age of group I was 65.0 years (range, 60 to 71 years). In group II, the mean age was 63.5 years (range, 56 to 69 years) and the mean follow-up period was 5.0 years (range, 3.1 to 9.1 years). No significant difference was found between the 


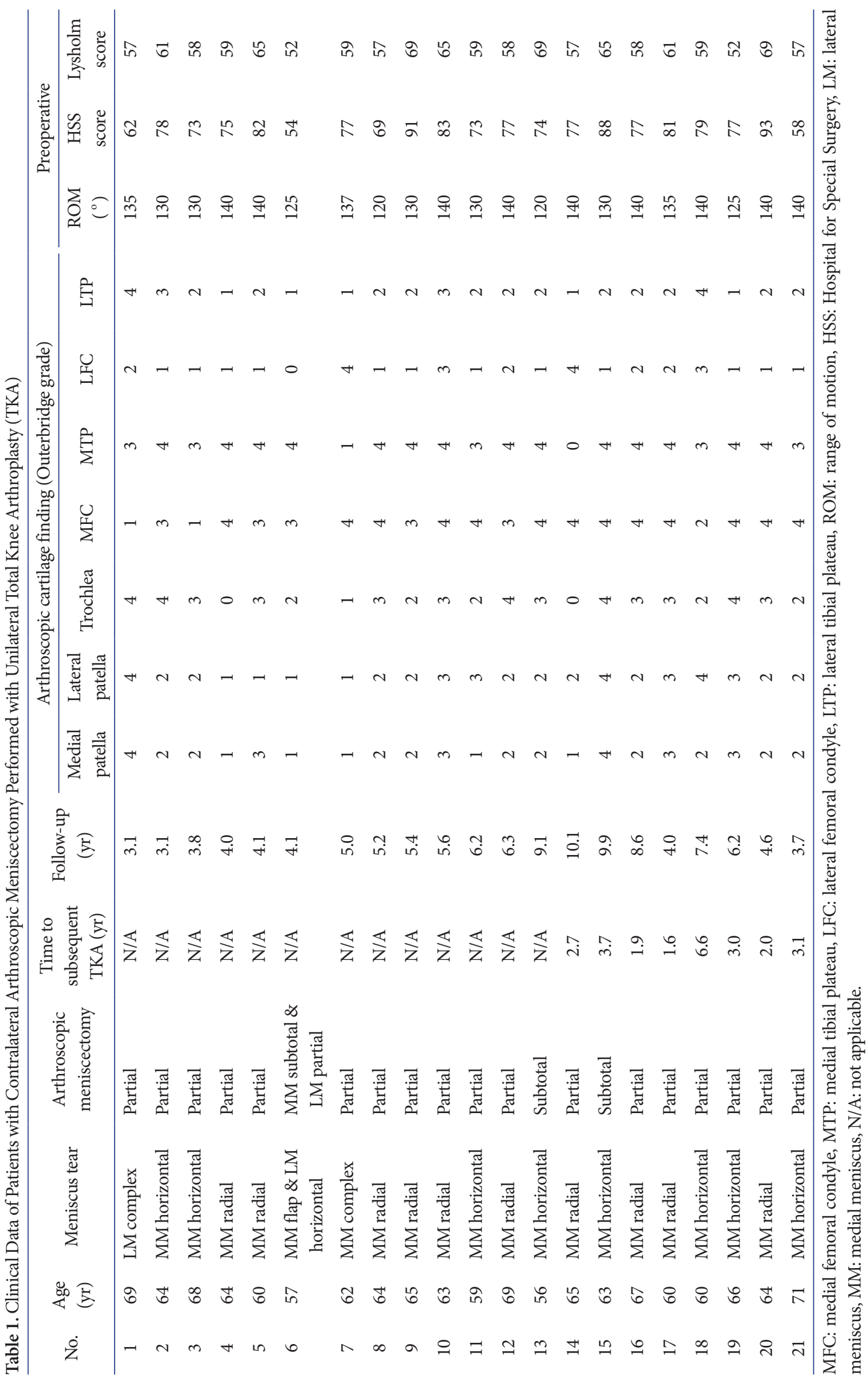


Table 2. Demographics and Preoperative Data of Group I and Group II

\begin{tabular}{|c|c|c|c|}
\hline Variable & Group I ( $\mathrm{n}=8)$ & Group II (n=13) & p-value \\
\hline Age (yr) & $65.0 \pm 3.3$ & $63.5 \pm 3.5$ & 0.411 \\
\hline Interval from initial meniscectomy to subsequent TKA (yr) & $3.1 \pm 1.5$ & - & \\
\hline Mean follow-up period (yr) & - & $5.0 \pm 1.6$ & \\
\hline Range of motion $\left({ }^{\circ}\right)$ & $136.3 \pm 5.4$ & $132.1 \pm 7.0$ & 0.188 \\
\hline HSS score & $78.8 \pm 9.6$ & $74.5 \pm 8.9$ & 0.335 \\
\hline Lysholm score & $59.8 \pm 4.9$ & $60.6 \pm 4.8$ & 0.710 \\
\hline \multicolumn{4}{|l|}{ Meniscus tear } \\
\hline MM horizontal & 4 & 4 & $1.000^{\mathrm{a})}$ \\
\hline MM radial & 4 & 6 & \\
\hline MM complex & 0 & 1 & \\
\hline LM complex & 0 & 1 & \\
\hline MM flap \& LM horizontal & 0 & 1 & \\
\hline
\end{tabular}

Values are presented as mean \pm standard deviation or number.

TKA: total knee arthroplasty, HSS: Hospital for Special Surgery, MM: medial meniscus, LM: lateral meniscus.

${ }^{\text {a) }}$ Fisher's exact test.

Table 3. Preoperative and Final Follow-up Clinical Data of Group II

\begin{tabular}{lccc}
\hline \multicolumn{1}{c}{ Variable } & Preoperative & Final follow-up & p-value \\
\hline ROM $\left({ }^{\circ}\right)$ & $132.1 \pm 7.0$ & $129.6 \pm 11.0$ & 0.566 \\
HSS score & $74.5 . \pm 8.9$ & $90.8 \pm 5.4$ & $<0.001$ \\
Lysholm score & $60.6 \pm 4.8$ & $82.4 \pm 4.6$ & $<0.001$ \\
\hline
\end{tabular}

Values are presented as mean \pm standard deviation.

ROM: range of motion, HSS: Hospital for Special Surgery.

groups in age at index operation $(\mathrm{p}=0.411)$, preoperative ROM (136.3 $\pm 5.4,132.1 . \pm 7.0 ; \mathrm{p}=0.188)$, preoperative HSS knee score (78.8 $\pm 9.6,74.5 . \pm 8.9 ; \mathrm{p}=0.335)$, and the Lysholm knee score $(59.8 \pm 4.9,60.6 . \pm 4.8 ; \mathrm{p}=0.710)$ (Table 2$)$. The distribution of meniscal tear type was not significantly different between groups $(\mathrm{p}=1.000)$. In group II, the mean preoperative and postoperative ROM (132.1 $\pm 7.0,129.6 . \pm 11.0)$ showed no significant difference $(\mathrm{p}=0.566)$ and the HSS knee score $(74.5 . \pm 8.9,90.8 . \pm 5.4)$ and the Lysholm knee score $(60.6 \pm 4.8,82.4 \pm 4.6)$ were improved significantly ( $\mathrm{p}<0.001$, respectively) (Table 3 ).

\section{Discussion}

The major finding of this study is that contralateral meniscectomy simultaneously performed with unilateral TKA showed relatively good results regardless of cartilage injury.

There are several studies on the progression of contralateral knee osteoarthritis after primary unilateral knee arthroplasty. Ritter et al. ${ }^{1)}$ reported that the probability of undergoing a subse- quent arthroplasty by 7 years after index surgery in contralateral normal knees and contralateral knees with osteoarthritis was 5\% and $21 \%$, respectively. They also reported that the probability of needing a TKA in patients with contralateral knee osteoarthritis was increased to $37 \%$ at 10 years. In the present study, 8 of the 21 patients (38\%) had a subsequent TKA surgery by 6.6 years after initial operation and this finding is similar to that of the study of Ritter et al. ${ }^{1)}$. Mont et al. ${ }^{2)}$ also reviewed the history of the contralateral knee in patients who underwent unilateral primary total knee arthroplasty. According to the study, 93\% of the patients who had moderate or severe symptoms and severe radiographic arthritis of the contralateral side with Ahlback grading scale later underwent subsequent TKA. In contrast, patients who initially presented with mild symptoms or no symptom had only $9 \%$ incidence of subsequent TKA. In addition, McMahon and Block ${ }^{3)}$ reported that the baseline Kellgren-Lawrence (K-L) grade of the contralateral knee at the time of index surgery was a strong predictor of eventual contralateral $\mathrm{TKA}^{7)}$. In the study, the mean survival time to contralateral arthroplasty was similar between patients with K-L grade II and those with grade III, 131.7 months and 127.6 months, respectively. However, patients with K-L grade IV had a mean survival time of only 80.45 months, whereas no patients with grade 0 or I progressed to subsequent TKA. Sayeed et al. ${ }^{8)}$ also described that the 10-year probability of having a contralateral TKA after index TKA was 36\% and the probability increased to $70 \%$ when grade 4 of K-L grade radiographic changes were present. To sum up, two previous studies mainly used radiographic findings such as K-L grade or Ahlback scale to evaluate 
the risk factors of eventual subsequent TKA and considered these radiographic changes as strong correlation factors ${ }^{7,99}$.

However, our study showed different results from previous studies. In consideration of excluding the cases where obliteration of the joint space was observed on standing radiography, the knees of the present study might belong to Ahlback grade I or K-L grade I or II. Therefore, under the same K-L grade or Ahlback scale, the mean survival time to subsequent TKA was longer in previous studies than the present study. This difference might be attributed to the following reasons that previous studies included the cases with or without meniscal or cartilage lesions whereas all cases of the present study had a meniscal tear and cartilage injury even though the K-L grade or Ahlback scale was equally good. Hart and Spector ${ }^{10)}$ reported that the K-L grading scale is subjective in nature and excessively emphasizes osteophytes. In addition, the K-L grade has been known to be inadequate for detecting early articular cartilage loss ${ }^{11,12}$. To our knowledge, no other study to date has investigated the prognosis of meniscus or cartilage lesion of the contralateral knee after unilateral TKA. Considering the limitation of radiographic evaluation, the present study based on arthroscopic findings including Outerbridge grade is more meaningful than the former studies. In addition, quite a few osteoarthritis patients with Outerbridge grade 3 or 4 cartilage changes of this study had relatively good results at the final follow-up after arthroscopic meniscectomy performed simultaneously with contralateral TKA, and only 8 of 21 patients consequentially had a subsequent TKA. In consideration of the meniscus tear and cartilage injury, these results can be regard as relatively good compared with those of former studies.

In recent randomized controlled trials assessing the benefit of arthroscopic partial meniscectomy, Yim et al. ${ }^{13)}$ found that arthroscopic surgery was not superior to strengthening exercise alone and Sihvonen et al. ${ }^{14)}$ also reported that there were no significant differences between arthroscopic meniscectomy and sham surgery in terms of relief in knee pain or functional improvement of the knee. However, these studies included only the patients showing minor degenerative changes on the radiograph (K-L grade 0 or 1 ) and degenerative horizontal tears of the medial meniscus, and the patients having chondral injury were excluded. On the contrary to these two trials, the present study included not only horizontal tears of the medial meniscus but also radial tears and complex tears of the medial or lateral meniscus. Considering that the patients included in the present study had various types of meniscal tears and Outerbridge grade 3 or 4 osteoarthritis, conservative treatment is not considered as a suitable care. In a previous research, we reported that meniscal tear symptoms in patients with Outerbridge grade IV osteoarthritis can be improved using arthroscopic medial meniscectomy when performed with proper indications such as good alignment and definite meniscal symptoms and signs ${ }^{5}$. The present study also showed the corresponding outcomes even after simultaneous contralateral TKA.

Maxwell et al. ${ }^{15)}$ reported that increasing levels of pre-arthroplasty contralateral knee pain is strongly associated with an increased prevalence of poor post-arthroplasty function and slow walking speed. They also mentioned that patients with unilateral knee arthroplasty and contralateral knee pain had a higher likelihood of poor functional outcomes compared with those with bilateral knee arthroplasty. In this respect, it is considered that contralateral knee pain should be evaluated and cured initiatively to have better prognosis of unilateral TKA. On the contrary, because load on the contralateral knee usually shifts to the more recovered arthroplasty-performed knee with time, even if the status of the contralateral knee may be worsened initially with increased load during surgical recovery, it is assumed that TKA have a positive effect on the prognosis of the contraleteral meniscectomized and cartilage-damaged knee in the present study.

The present study suggests that arthroscopic meniscectomy of the contralateral knee carried out simultaneously with unilateral TKA would be effective in spite of Outer-Bridge grade 3 or 4 cartilage degeneration if performed with exact and proper indications, such as more predominant mechanical meniscal pain and relative good alignment. The limitations of this study include the small number of study population, short follow-up period, retrospective study design, and lack of a comparison group.

\section{Conclusions}

Contralateral arthroscopic meniscectomy performed simultaneously with unilateral TKA provides relatively good results regardless of cartilage degeneration in the patients with good knee alignment and definite mechanical meniscal symptoms.

\section{Conflict of Interest}

No potential conflict of interest relevant to this article was reported

\section{References}

1. Ritter MA, Carr KD, Keating EM, Faris PM. Long-term outcomes of contralateral knees after unilateral total knee 
arthroplasty for osteoarthritis. J Arthroplasty. 1994;9:347-9.

2. Mont MA, Mitzner DL, Jones LC, Hungerford DS. History of the contralateral knee after primary knee arthroplasty for osteoarthritis. Clin Orthop Relat Res. 1995;(321):145-50.

3. McMahon M, Block JA. The risk of contralateral total knee arthroplasty after knee replacement for osteoarthritis. J Rheumatol. 2003;30:1822-4.

4. Bin SI, Kim JM, Shin SJ. Radial tears of the posterior horn of the medial meniscus. Arthroscopy. 2004;20:373-8.

5. Bin SI, Lee SH, Kim CW, Kim TH, Lee DH. Results of arthroscopic medial meniscectomy in patients with grade IV osteoarthritis of the medial compartment. Arthroscopy. 2008;24:264-8.

6. Outerbridge RE. The etiology of chondromalacia patellae. J Bone Joint Surg Br. 1961;43:752-7.

7. Kellgren JH, Lawrence JS. Radiological assessment of osteoarthrosis. Ann Rheum Dis. 1957;16:494-502.

8. Sayeed SA, Sayeed YA, Barnes SA, Pagnano MW, Trousdale RT. The risk of subsequent joint arthroplasty after primary unilateral total knee arthroplasty, a 10-year study. J Arthroplasty. 2011;26:842-6.

9. Ahlback S. Osteoarthrosis of the knee: a radiographic investigation. Acta Radiol Diagn (Stockh). 1968:Suppl 277:7-72.

10. Hart DJ, Spector TD. Radiographic criteria for epidemiolog- ic studies of osteoarthritis. J Rheumatol Suppl. 1995;43:46-8.

11. Brandt KD, Fife RS, Braunstein EM, Katz B. Radiographic grading of the severity of knee osteoarthritis: relation of the Kellgren and Lawrence grade to a grade based on joint space narrowing, and correlation with arthroscopic evidence of articular cartilage degeneration. Arthritis Rheum. 1991;34:1381-6.

12. Schiphof D, Boers M, Bierma-Zeinstra SM. Differences in descriptions of Kellgren and Lawrence grades of knee osteoarthritis. Ann Rheum Dis. 2008;67:1034-6.

13. Yim JH, Seon JK, Song EK, Choi JI, Kim MC, Lee KB, Seo HY. A comparative study of meniscectomy and nonoperative treatment for degenerative horizontal tears of the medial meniscus. Am J Sports Med. 2013;41:1565-70.

14. Sihvonen R, Paavola M, Malmivaara A, Itala A, Joukainen A, Nurmi H, Kalske J, Jarvinen TL; Finnish Degenerative Meniscal Lesion Study (FIDELITY) Group. Arthroscopic partial meniscectomy versus sham surgery for a degenerative meniscal tear. N Engl J Med. 2013;369:2515-24.

15. Maxwell J, Niu J, Singh JA, Nevitt MC, Law LF, Felson D. The influence of the contralateral knee prior to knee arthroplasty on post-arthroplasty function: the multicenter osteoarthritis study. J Bone Joint Surg Am. 2013;95:989-93. 